\title{
Problems of Social Ecology and their Specific Character in the Kaliningrad Region
}

\author{
Galina M. Barinova*
}

\author{
Eduard S. Demidenko
}

Evgeniy V. Krasnov

Immanuel Kant Baltic Federal University, Russian Federation; *Corresponding Email: GBarinova@kantiana.ru

\section{Doi:10.5901/mjss.2015.v6n6s7p26}

\section{Abstract}

The study touches upon the issues of social ecology that arise in the westernmost region of Russian Federation - the Kaliningrad region. The specificity of the geographical location of this subject of the Russian Federation, being defined by its exclave positioning, creates specific development features, which inevitably influence all spheres of well-being. Papers reflects on research results of a survey held on evaluating the environmental and economic situation in the region. Results suggest that little attention paid to the problems of social ecology in the region, while the policy documents pay minor attention to the environmental status of the territory. Having analysed the previously elaborated models on the ways of transition to environmentally sound development, authors propose a balanced natural resources management approach.

Keywords: Kaliningrad region, social ecology, sustainable development

\section{Introduction}

Social security as well as many other aspects of social activity has a certain regional dimension related to the historical, geopolitical, environmental, cultural and other features of a local community life. In this regard, we cannot leave aside the specific manifestations of social security in such a region as the Kaliningrad region (Barinova \& Krasnov, 2006; Mikhaylova, 2015). Being an integral part of Russia, the region at the same time is a kind of double periphery - on the one hand, it is an enclave within the European Union, on the other hand, it is the only overseas territory of the Russian Federation, its exclave. Such a situation arose in the early 1990s after the collapse of the USSR when the region was cut off from the rest of the country overnight. Naturally, all this could not help affecting, both objectively and subjectively, the social security of the regional community.

Among the objective factors are complications in connection and interaction between the residents of the region and their relatives living in the mainland Russia; deterioration of cargo transit conditions, both to and from the territory of the region; possible difficulties in supply of electricity, natural gas and other material resources (Krasnov \& Krivosheev, 2006). It is clear that the construction of a thermal power plant and the beginning of construction of a nuclear power plant are intended to secure energy supply to the region, but this is only one aspect of security of the region.

These and many other objective conditions of security affect the social well-being of the population in the region. In 2016, the consequences of the effect of the new Federal Law on free economic zones will affect the region to the full, which may cause a landslide reduction of staff in many industries that are currently enjoying certain preferences and privileges. It constantly keeps the regional community in tension and does not contribute to the growth of the Kaliningrad residents' confidence in the future. To some extent, this tension reflects the environmental problems of the region as well.

\section{Socio-ecological Situations of Stress}

The degree of tension in the situation of social stress in the Kaliningrad region with its high density of population (over 60 people per sq. km.) in towns and cities is much higher than in the rural areas, which is largely due to building density, transport density (with its inevitable 'traffic jam'), excessive amount of motor vehicles and other negative environmental urban processes. According to the Kaliningrad Regional Committee of Statistics, in seven cities of the region, except for the city of Kaliningrad, the level of emission of pollutants into the atmosphere reaches 220-230 thousand tons. At the same time, it should be noted that emissions from stationary sources are decreasing and those from transport are increasing up to $83-86 \%$ of the total. 
The Kaliningrad region is one of the leaders in Russia in terms of the number of motor vehicles. These are mainly used cars with low performance and technical characteristics, which contribute significantly to air pollution. Air quality in Kaliningrad, Chernyakhovsk and other settlements is found to be unsatisfactory with high levels of pollution from year to year.

The problem of industrial and household waste disposal, including recycling and dumping, is not less complicated. There are more than 160 dumpsites for municipal solid waste with the total area of 140 hectares. The total volume of industrial and household waste accounts for 35.0 million $\mathrm{m}^{3}$. The greatest damage to water bodies is caused by the discharge of untreated industrial and household wastewater. Most of the contaminants (chlorides, sulfates, ammonia nitrogen, phosphorus, methanol, formaldehyde and others) go directly into the Baltic Sea or the Curonian and Vistula lagoons and into the numerous surface watercourses and groundwater.

That is why providing the population with safe drinking water has been one of the socially important environmental issues in the region for many decades. Basing on the public reports "On the sanitary and epidemiological situation in the Kaliningrad region" certain correlation between the morbidity rate and environmental pollution has been observed (Statisstical digest, 2013; Krasnov et al., 2007). For example, when analyzing the incidence of the diseases of musculoskeletal and genitourinary systems, a direct correlation between those and the iron content in drinking water in the Svetlogorsk municipality $(r=0.91-0.94)$ and the Ozersk municipality $(r=0.69-0.73)$ was observed.

As for the structure of morbidity, the first place has been occupied by respiratory diseases for a long time, which reflects a poor quality of air. About 83,000 people (mainly in the city of Kaliningrad) are subject to the effects of high concentrations of nitrogen dioxide and suspended matter in the atmosphere. A clear correlation between respiratory diseases and the contamination of air with formaldehyde has been established as well $(r=0.83)$.

In order to assess the quality of life and level of social stress in the region it was offered to use the integral index of well-being (i). It is calculated for each administrative district or municipality as the ratio of normalized statistical indicators: average monthly wage (a), the number of beds in hospitals (b), living space in cubic meters (c), the number of the unemployed (d), the number of crimes (e) and other forms of deviant behavior (Krivosheev, 2010; Krivosheev, 2012).

$i=a+b+c / d+e^{\star} 100 \%$

The value of the index of well-being of 70-100\% represents the most favorable conditions for the population.

\section{Quality of Life}

The issue of the quality of life in the region is discussed from a different angle, namely to identify how the residents of the Kaliningrad exclave understand the real environmental and economic situation. In order to find out about this, sociologists and environmentalists from Immanuel Kant Baltic Federal University conducted a survey in the community (Barinova \& Krasnov, 2006). The entire adult population (18 years and older) permanently living in the region was considered to be general population. The sample size was 1,000 people ( $+6 \%$ excess of the selection); the sample type was three-stage with quoting the respondents by sex, age and education, with a random selection of respondents at the study point. The survey was carried out in 15 cities, including the regional center, and 20 rural settlements. A formalized questionnaire was used as a tool (see Table 1).

Table 1. Factors determining the quality of life

\begin{tabular}{lc}
\hline Quality of life factors & Percentage of respondents \\
\hline Financial well-being & $58.8 \%$ \\
Health & $47.2 \%$ \\
Quality of medical services & $43.6 \%$ \\
Accommodation & $38.0 \%$ \\
Absence of unemployment & $19.0 \%$ \\
Education level & $17.5 \%$ \\
Personal security & $17.3 \%$ \\
Environmental conditions & $13.9 \%$ \\
Good nutrition & $12.2 \%$ \\
Opportunities for good rest & $5.7 \%$ \\
Other & $1.7 \%$ \\
No answer & $3.0 \%$ \\
\hline
\end{tabular}

Note: the total percentage is more than $100 \%$ because the respondents could choose up to three factors determining the quality of life. 
Among the factors determining the quality of life, the leading one is the financial well-being, the second and third places are taken by health and quality of medical services, and the fourth by accommodation issues. Such factors as the level of education, personal security, and environmental conditions are of less importance.

The majority of the respondents link the very low health self-assessment to the little attention paid to the problems of social ecology and personal unawareness of the correlation between living standards and the quality of one's own life. Thus, more than half (53.2\%) of the respondents believe that the health of Kaliningrad residents is unsatisfactory.

The residents of Kaliningrad evaluate environmental conditions as bad (22\%) and very bad (35\%). The residents of rural areas are more optimistic in their evaluation of the situation $-49 \%$ of the respondents identified these conditions as good or even very good.

Instead of a purely economic or consumer approach to the regional development strategy with policy documents, at best, having a small section devoted to the environmental status of the territory, it was proposed to take a socioecological approach as a basis for government (Krasnov et al., 2007). The priority objectives for development should include real improvements in education, healthcare, raising environmental awareness and culture, biodiversity preservation, recreation development, etc.

Taking into account regional specificity, the key requirements for the concept of a more sustainable development of the Kaliningrad region are the following:

- the crisis situations of economic and environmental character should be overcome in their interconnection considering the objectives of sustainable development of the community;

- aggravated external conditions should be taken into account, especially the concentration of the armed forces of NATO and the EU close to the borders of Russia including our region;

- enclave/exclave position should be used for the benefit of the region by enhancing international cooperation in all spheres - economic, social and environmental;

- sectoral priorities of economic development should more completely reflect the internal natural and resource potential of the region (deposits of oil, amber and other natural resources, recreational opportunities, etc.), which can provide not only stabilization but also a significant improvement in the environmental, economic and, consequently, social situation;

- regional development is intended to ensure a radical improvement of the socio-environmental situation through cleaner production, science and education.

It is extremely important for the Kaliningrad region to have really applicable environmental legislation, particularly with regard to environmental performance of vehicles, traffic and movement of people.

\section{Environmental Policy and Regional Legislation}

Environmental safety of motor vehicles is one of the priorities in solving regional problems. The contribution of motor vehicles into the total volume of air pollution is steadily growing. Certain measures to reduce the harm from exhaust fumes are taken at various stages: when manufacturing engines and motor fuel, when crossing the customs border, in urban planning, development and construction, etc.

However, despite some achievements, such as the introduction of special technical regulation standards 'Euro 5' and the prohibition of production and circulation of leaded motor gasoline (Federal Law of March 22, 2003 №34-FZ), it has not been possible to change the negative trend yet.

In some regions, local legislation is initiated to regulate these issues and local authorities actively use their powers to restrict movement of vehicles in towns, places of recreation and tourism; they limit the use of fuel that after combustion leads to air pollution, encourage production and use of environmentally friendly fuels. However, the purview of regional legislative bodies is limited by the spheres of joint competence with the federal authorities. Keeping in mind that in addition to the exhaust emission and noise pollution the exploitation of vehicles leads to other negative consequences (for example, the seizure of new land for broadening roads and the construction of parking lots, cluttering areas with abandoned old cars) and affects, besides environmental protection, many other kinds of relations, in particular the property rights, freedom of movement, business, traffic, all these are prerequisites for the integrated regulation of environmental safety of motor vehicles. To improve the environmental situation, new taxes should be imposed on car owners (as an option, on emissions or fuel, on the movement in the city center), which should be differentiated according to environmental classification of vehicles. Even though any additional charges are not popular among the population, sooner or later the methods of economic incentives to improve the environmental performance of vehicles and to reduce vehicle trips will be applied for the solution of the problem of exhaust reduction. As a direct restriction (the ban on the 
purchase and use) is not possible, no other more effective measures than increased cost of car usage have been found yet. The adoption of more strict standards is justified by paramount importance of the right to life and good health. Important tasks should also include the protection of the environment from pollution by industrial and household waste. Their solution involves reducing the volume of waste, safe disposal and use as secondary material resources. The registration and circulation of rights to toxic gases emission, trading in the quota market and the distribution of benefits should be legally regulated as well.

The incident of contamination of water bodies and watercourses from adjacent territories confirms the importance of the region's participation in monitoring the implementation of international agreements aimed at preventing crossborder pollution of water and other natural objects, as well as those in which Russia is not a party yet (in particular, invigorating the activities of non-governmental environmental organizations is necessary). Environmental legislation containing environmental and natural resource use norms as well as dozens of laws and thousands of regulations is quite voluminous and difficult to use.

One of the ways to organize and systematize the regulation of environmental legal relations is the codification of legislation. The most important condition of the codification is the inventory check of the relevant laws, increasing their efficiency, elimination of inconsistencies, identifying objective necessity to make new laws. There is a need in a wideranging discussion of the draft concept of the Environmental Code of Russia developed by the Ministry of Natural Resources and Environment. Certain attention should be paid to the experience of combating with and prevention of environmental violations gained by some environmental prosecutor's offices and police departments. However, there is a need to improve the legal framework of their activities in order to transfer it from an experimental status into a stable mode. Administrative and procedural powers of internal affairs bodies could be expanded by granting them the right not only to draw up protocols (Par. 1 Part 2, Art. 28.3 of the Administrative Code) but also to investigate the administrative cases of environmental offences followed by relevant decrees on a wider range of offences than stipulated in the Code of Administrative Offences.

The process of establishing legal mechanisms of compensation for environmental damage to health is at a standstill. General civil laws hardly ever work in such cases due to many different peculiarities of such damage, its causes and manifestations, while the Federal Law on Environmental Protection does not regulate any sectoral environmental liabilities; as a result, the number of cases won in courts is negligible. However, the reason for that is not only conservatism of the legislative bodies and the inertia of the victims themselves, who speaking actively and massively in defense of their right to good health protected from exposure to negative environmental factors could thus make a social demand for the adoption of relevant regulations, but also objective difficulties related to insufficiently grounded scientific recommendations. The best way to tackle many issues of social ecology could be the following. First, an extensive and sufficient for calculations and conclusions database of socio-environmental monitoring should be established, which would serve as an information basis for the application of traditional methods of bringing perpetrators to justice and health risk assessment in terms of negative environmental factors. It is obvious that now there are not enough data of socio-hygienic and environmental monitoring for making confident judgments about the degree of environmental causes for diseases.

Economic assessment of environmentally caused morbidity is extremely important. For the time being there are only a few methods that, as a rule, use foreign algorithms. The approach to assessing the damage from environmentally caused morbidity has not fundamentally changed since the late 1980s. The following factors were taken into account in calculations carried out in some regions: the cost of the state health insurance system for the treatment of population; the cost of purchasing medicines and visiting a doctor by the population; the cost of health resort treatment; sick pays; the loss of gross regional product.

All this gives us hope that in the near future a complete framework for the development of normative legal acts will be created. Unfortunately, scientific research of the influence of negative environmental factors on human health lags far behind the needs.

One of the most important issues of providing energy supply security and environmental security is that of energy saving, which can drastically reduce the use of non-renewable natural resources and save up to $50 \%$ of irrationally used energy resources. It is known that in specific ratio Russia spends many times more energy than, for example, European countries, even after adjusting for environmental conditions. Therefore there is a need in urgent analysis of the causes of the low efficiency of the legislation on energy consumption, in particular of the Federal Law "On energy saving". In order to save energy, there are well known and, as international experience shows, reliable alternative energy sources such as solar, geothermal, and bioenergy. 


\section{Ways of Transition to Environmentally Sound Development}

The transition to a sustainable development model (according to the decisions of the UN Conference on the environment, Rio de Janeiro, 1992) provides a widespread use of new technologies, renewable energy, restoration of natural landscapes (revitalization of deserted and cluttered lands), expansion of international economic integration (globalization), etc. Less popular model of transition is that of 'social and environmental post-industrialism', which involves a decrease in population and production growth, the rejection of a unified model (American style) of the world, the preservation of ethnic and cultural diversity of countries and regions to achieve regional self-sufficiency in food and commodities. Another way of transition to a more balanced and sustainable development is associated with 'environmental industrialism', which is understood as an improvement of traditional technologies and industries, energy and resource saving, economizing on raw materials and energy consumption per unit of output (and as a consequence, reducing waste including toxic pollutants).

Entire economic regions and subjects of the Federation or their parts with different combinations of ethno-cultural, demographic, and economic development opportunities are sometimes presented as 'safety islands'. Of course, this approach does not put aside other concepts, including inter-regional and even global cooperation, 'cross-border clustering' (e.g. see Mikhaylov, 2015), etc.

In the context of the Kaliningrad region, the transition seems to be possible in accordance with the UN decisions. Balanced natural resources management in the context of Russia who possesses enormous natural resources, vast territory and talented multinational people is quite possible, which was repeatedly confirmed in the past by quick recovery of economy destroyed in wars (Krivosheev \& Danchenko, 2013).

\section{References}

Barinova, G.M., \& Krasnov, E.V. (2006). Socio-ecological realia and the strategy of the Kaliningrad region development. Territorial differentiation and regionalization in the modern world (pp. 41-45). Smolensk: Universum.

Federal Law of the Russian Federation "On energy saving" (1995)

Krasnov, E.V., \& Barinova, G.M., \& Krivosheev, V.V. (2007) Managing social anomie in Russia (geo-informational aspect). Sustainable development of areas: GIS theory and practical experience: Proceedings of International conference. V.1. Khanty-Mansiysk.

Krasnov, E.V.,\& Krivosheev, V.V.(2006). Social ecology: from science to reality. Sociological Studies. No.7, 93-96.

Krivosheev, V.V. (2012). Social security: general aspects and regional dimension. State and municipal service: status, experience, further mastering: collection of articles ( pp 84-91). Kaliningrad: Baltic Institute of Economics and Finance.

Krivosheev, V.V.(2010). Social security and social pathology: the facets of the conjugation of the concepts and phenomena. Immanuel Kant RSU Vestnik. V.12, 130-135.

Krivosheev, V.V., \& Danchenko, A.S. (2013) Social responsibility of public servants: results of the sociological research. Local government and municipal service personnel training: status and problems (pp 47-53). Kaliningrad: Baltic Institute of Economics and Finance.

Miagkov, S.M. (1995). Ways to socio-ecological sustainability of Russia. Moscow University Vestnik. Series 5. Geography, 3-9.

Mikhaylov, A.S. (2015). Conceptualizing international cluster. Mediterranean Journal of Social Sciences, 6 (3), 11-18.

Mikhaylova, A.A. (2015). Region in the national security system of Russia. Social and Economic Geography, 1(1), 9-15.

The Kaliningrad region healthcare in figures (2013). Statistical digest. Kaliningrad. 